\title{
A time-dependent decision support system for multi-attribute decision-making
}

\author{
Sixto Ríos-Insua, Antonio Jiménez and Alfonso Mateos \\ Department of Artificial Intelligence, School of Computer Science, Madrid Technical University, Campus de \\ Montegancedo $s / n$, 28660 Madrid, Spain \\ Tel.: +34913367437; Fax: +34913524819; E-mail: srios@fi.upm.es
}

\begin{abstract}
We describe a decision support system including multiple conflicting objectives and with consequences spanning several time periods. The system is supported by an imprecise multiattribute additive utility model, which includes the possibility of different discounting methods, aimed at identifying the optimal strategy by means of interactive multiobjective simulated annealing. The system can be useful for solving problems, including, if necessary, decisions with imprecise consequences over different time periods, which are modelled by means of an objective hierarchy. This objective hierarchy considers appropriate preferences based on judgemental inputs for assessing imprecise individual utility functions and imprecise scaling factors on objectives and attributes, which are aggregated into a scalar or vectorial additive utility function to evaluate the available alternative decisions. The system also includes different sensitivity analyses to check the robustness of the conclusions. Finally, an application to the evaluation of remedial strategies for restoring contaminated aquatic ecosystems illustrates the usefulness and flexibility of this decision support tool.
\end{abstract}

\section{Introduction}

The complexity of many real decision-making problems has recently led to the development of better and better decision support tools to deal with the increasing difficulties involved in these problems. The tools of this kind should be able to consider some of the basic aspects that arise in real problems, like the presence of multiple conflicting objectives spanning several time periods, as well as imprecision concerning the assignments.

To deal with such complex situations, we have developed a decision support system (DSS) that can construct an objective tree over time with up to $150 \mathrm{ob}-$ jectives, 10 objective levels and 10 time periods. The system is able to capture and deal with objective trees that model appropriate time imprecise preferences, i.e., it models preferences in a hierarchical structure over time periods by means of utility functions, taking into account the effects of discounting on the chosen outcomes. This approach can help DMs to express their opinions or judgements with a better understanding of the situation, because attributes, weights and the un- certainty surrounding them are allowed to change over time.

Several studies have been conducted on how DM preferences on objective and attribute weights change over time [2,10] and [17], showing that an objective's importance can change. This has to be reflected in the underlying model. So, the developed system assumes that attributes may be time-dependent and linked to the period in which they occur. In intertemporal decisions, trade-offs between the present and the future must be made explicit, and this can be considered by structuring the objective hierarchy in such a way that the attributes' importance is specified in each period. Thus, we propose dividing the hierarchy used to support the decisions with consequences into time periods, so that a different discount can be assigned to each attribute for each period. The attribute set may be the same for each time period, but one can imagine many circumstances where they differ. In this case, different attribute sets describing the DM's perceptions of value would be considered in the different periods. For ease, we assume that the attribute set for each time period $t$ is the same. Therefore, the DM will assign a weight 
equal to zero to any fixed or given attribute that is not relevant in a particular time period, and its contribution is then null. This is implemented in the system by means of attribute activation/deactivation. Figure 1 shows a diagram of such a hierarchy. Intertemporal decisions could be considered as an explicit trade-off between the present and the future by structuring the objective tree so that the importance of the significant attributes in each period is made explicit.

The DSS is based on an additive multiattribute utility model [16], which allows for imprecision concerning the inputs [21] and [24], and partial information of the DM's preferences. Thus, the process of assessing the individual utility functions and the constant scales is not very demanding, which is less stressful on DMs.

Furthermore, we shall consider the situation where the consequences might have some uncertain policy effects, as considered in [18]. So, the system will also allow for uncertainty about the consequences or outcomes, where they can be entered as ranges or intervals instead of single values as an approach under certainty would demand. Note that this more general viewpoint leads to a more robust approach to decision making, which can account for imprecision and could overcome some of the criticisms of Decision Analysis.

Thus, the starting point will be to establish a set of $n$ attributes denoted by $X_{1}, \ldots, X_{n}$ and the time periods $t=1, \ldots, T$. Then, the consequence of each strategy or alternative decision $S^{q} \in \mathrm{S}$, where $S$ is the available strategies set, is a stream defined by a vector of intervals

$$
\begin{aligned}
& \left(\left(\left[x_{1 q}^{L 1}, x_{1 q}^{U 1}\right], \ldots,\left[x_{n q}^{L 1}, x_{n q}^{U 1}\right]\right), \ldots,\left(\left[x_{1 q}^{L t}, x_{1 q}^{U t}\right], \quad(1)\right.\right. \\
& \left.\left.\left(\left[\ldots,\left[x_{n q}^{L t}, x_{n q}^{U t}\right]\right), \ldots, x_{1 q}^{L T}, x_{1 q}^{U T}\right], \ldots,\left[x_{n q}^{L T}, x_{n q}^{U T}\right]\right)\right)
\end{aligned}
$$

where $x_{i q}^{L t}$ and $x_{i q}^{U t}$ are, respectively, the lower $(L)$ and upper $(U)$ endpoints of the imprecise consequence for attribute $X_{i}$ in the time period $t$. We assume that there is a continuous uniform distribution over each interval $\left[x_{i q}^{L t}, x_{i q}^{U t}\right]$, and both endpoints being equal, i.e., $x_{i q}^{L t}=x_{i q}^{U t}$, would be equivalent to the case under certainty, where the policy effects for a strategy $S^{q}$ in attribute $X_{i}$ for time period $t$ are precisely known. In any case, we also consider the possibility of substituting each interval by a single value given by the average $x_{i q}^{P t}=\left(x_{i q}^{L t}+x_{i q}^{U t}\right) / 2$ ( $P$ means precision $)$, having then a precise consequence, if deemed appropriate by the DM.

Next, an analysis focused on judgemental inputs must be conducted to assess imprecise utility functions on attributes and imprecise scaling factors or weights on objectives and attributes in the objective tree, and for their aggregation into a global utility function that includes, where appropriate, discount factors for each attribute set to evaluate timestreams, as we shall explain below. The methodology has been implemented on a PC-based DSS, where all process-relevant information can be entered to help DMs arrive at the best or a satisfactory strategy. Finally, a multiparametric sensitivity analysis is introduced to check the sensitivity of conclusions on the inputs.

The following deals with the multiattribute utility modelling process under imprecision. Section 3 considers different discounting methods for providing DMs with assistance concerning how to tradeoff present and future. Due to the difficulty in determining the best strategy for problems with a large number of them, an approximation approach is proposed in section 4, supported by a optimization methodology based on interactive multiobjective simulated annealing, which is described in section 5. Section 6 outlines multiparametric sensitivity analysis. In section 7 , we show the final model formulation illustrated with an example on restoring aquatic ecosystems and, finally, some conclusions are provided.

\section{A procedure for assessing the imprecise multiattribute utility function}

Expected multiattribute utility theory can be considered as a leading paradigm for normative decision theory. However, multiattribute utility theory calls for the DM to provide all the information describing the decision situation to assess component scalar utility functions $u_{i}[6,25]$, and [28]. This can be far too strict in most practical situations, which could lead to the consideration of imprecise component utility functions, see [23] and [30].

In this modelling context, what would be called imprecise timestream utility efficient strategy set plays an important role, because it has a similar property to the well-known efficient or Pareto optimal strategy set: given a strategy in this set there is no other strategy in $S$ that dominates it. However, this set can be difficult to determine in this new context, so intelligent approaches are needed to help the DM to arrive at a final solution, as we shall see later.

Now, note that it is necessary to assess a scalar utility function $u_{i}$ for each attribute $X_{i}$, that reflects DM preferences on the possible attribute values. The drawbacks associated with utility function assignment are well known, even though good software is available for 


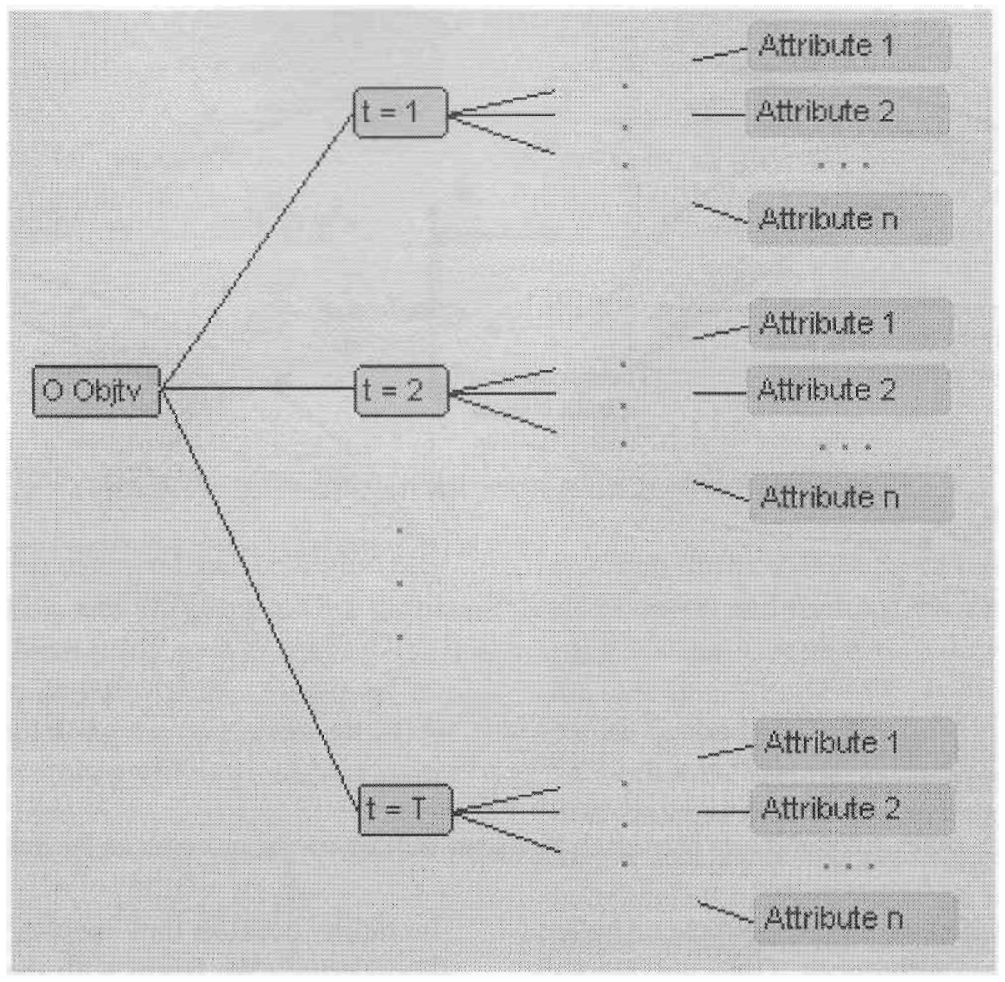

Fig. 1. Diagram of an objective tree including time periods.

this process. Several authors, see, e.g. [12,13] or [19], have suggested that elicited utility functions are generally method dependent, and bias and inconsistencies may be generated in the assignment process. Our system attempts to overcome these objections by combining two slightly modified standard procedures: the fractile method and the extreme gambles method [5], which belong to the certainty equivalence (CE) and probability equivalence (PE) categories, respectively. Moreover, it assumes imprecision allowing the DM to provide a range of responses instead of a precise value, as the above methods demand for each probability question faced by the DM. This is less stressful on experts, since they are allowed to provide incomplete preference statements, see [22] and [29]. As a result, we get a utility function class for each method rather than a single function. The intersection between the two classes provides the range where preferences elicited by the above methods agree. Should such intersections be empty for an interval, the DM would be inconsistent and his/her preferences should be reelicited. The process finishes when a consistent range is obtained.

Specifically, in the fractile method, see [5] and [16], the DM is asked to provide certainty equivalence intervals or attribute value intervals $\left[x_{i}^{L j}, x_{i}^{U j}\right]$ that he/she considers equivalent to different gambles, whose results are the most and least preferred attribute values $x_{i}^{*}$ and $x_{i *}$ with probabilities $p^{j}$ and $1-p^{j}$, respectively. We take $p^{1}=0.25, p^{2}=0.50$ and $p^{3}=0.75$. The method determines a range in the $x_{i} / u_{i}\left(x_{i}\right)$-diagram, which can be represented by the bounding utility functions $u_{i}^{L_{C E}}$ and $u_{i}^{U_{C E}}$, where $L(U)$ means Lower $(U$ pper). Figure 2 shows this utility function class drawn between dotted lines.

The system includes a routine implementing a wheel of fortune to output these probability intervals, see [8], which shows the probabilistic questions and guides the expert until an interval of indifference probabilities is obtained. A number of additional questions are included as consistency checks.

In the extreme gambles method, see [5] and [16], the DM has to specify probability intervals. We use the upper bounds of the certainty equivalent intervals provided by the DM in the CE-method as sure amounts. Other points may be used for comparison. Figure 2 also shows the utility function class drawn between solid lines and represented by the bounding utility functions $u_{i}^{L_{P E}}$ and $u_{i}^{U_{P E}}$, with $L$ and $U$ as above.

As mentioned above, should the intersection of both ranges be empty for some attribute values, the DM 


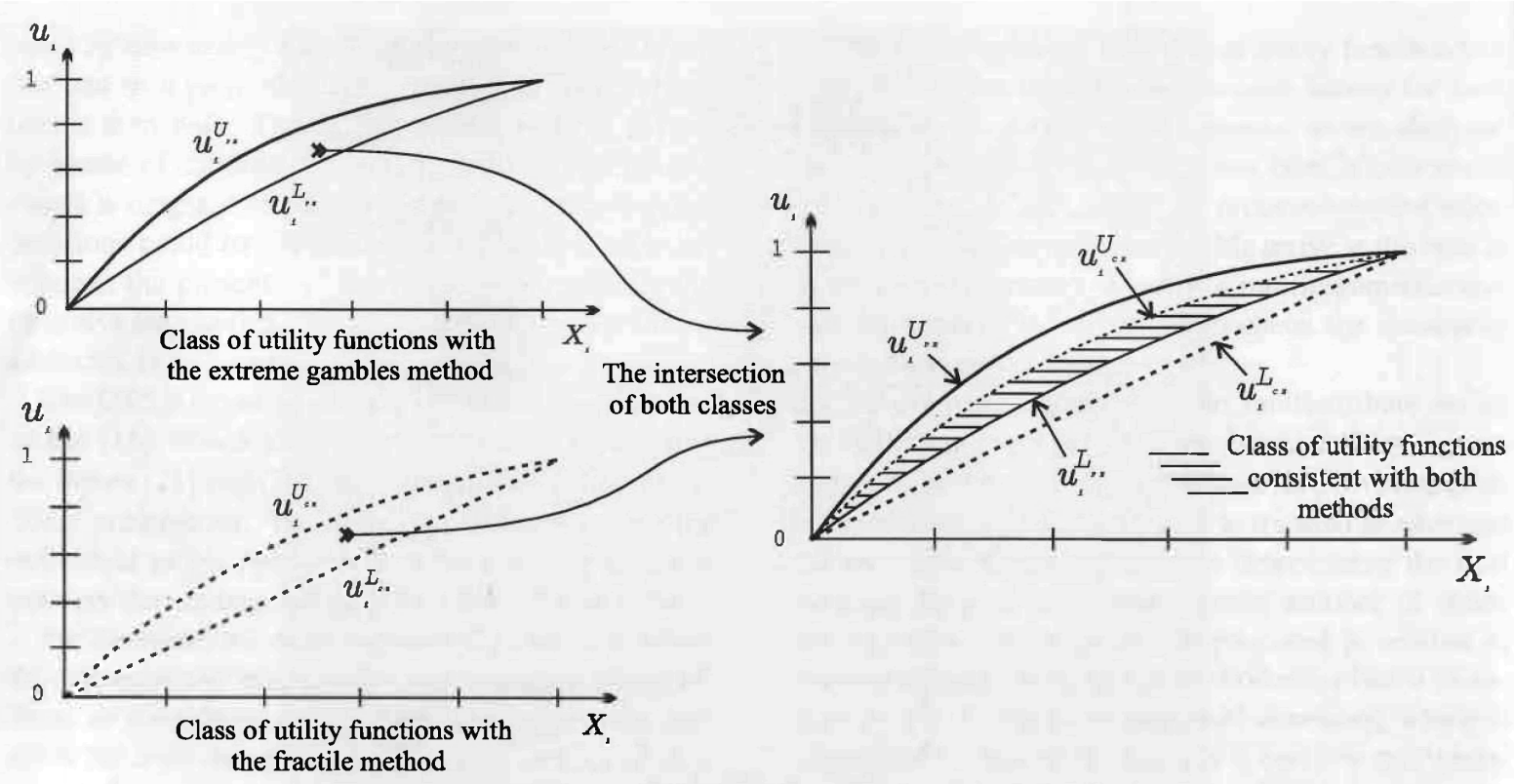

Fig. 2. Utility function classes of based on the fractile and extreme gambles methods for attribute $X_{i}$.

would have provided inconsistent responses and he/she should reassess his/her preferences. Thus, the intersection will be the range for the DM's utility functions. The system is able to detect possible inconsistencies and suggests what the DM should change to achieve consistency. Thus, given a precise consequence $x_{i}$ for attribute $X_{i}$, we have a utility interval $\left[u_{i}^{L}\left(x_{i}\right), u_{i}^{U}\left(x_{i}\right)\right]$ instead of a single utility $u_{i}\left(x_{i}\right)$. This is shown for increasing utility functions in Figs 2 and 3 by the striped area.

As we shall see below, the evaluation process calls for precise utility functions in the problem-solving process, so the system assesses fitted utility functions $u_{i}^{P}$ ( $P$ means precision) by natural cubic splines interpolation through the mid-points of the utility intervals in the intersection area for each $u_{i}$, see Fig. 3. The result for decreasing utility functions would be similar.

We also need to assess positive scaling constants or weights $k_{i}$ to add the separate contributions of the attributes and get the additive multiattribute utility function. The DSS includes two methods for assessing such weights for any objective of the hierarchy: using tradeoffs, see [16], and direct assignment. The first procedure is based on trade-offs among the respective attributes at the lowest-level objectives stemming from one and the same objective. The DM is asked to provide probability intervals such that he/she is indifferent between lotteries and sure consequences. This procedure is best suited for the lowest-level objectives in the hierarchy because they involve a more specific area of knowledge. We begin with the objectives at the lowest level of the hierarchy and then continue the assessment in ascending order through the hierarchy. As in the case of utility assessment, imprecise assignments by means of ranges or intervals $\left[k_{i}^{L}, k_{i}^{U}\right]$ are possible. This means that the DM will be able to perform a global sensitivity analysis, allowing intervention at any level of the objective hierarchy.

The second procedure is based on direct assessment and is, perhaps, more suitable for upper level objectives, because they can be more political. Here, the $\mathrm{DM}$ is asked to provide weight intervals $\left[k_{i}^{L}, k_{i}^{U}\right]$, as before. Note that when the system is run, the starting point is equally weighted objectives. However, any interval or precise weight can be changed, and the system automatically takes care of how these changes should be propagated through the objective hierarchy and recalculates the overall vector utility for each strategy.

In both assessment procedures, the system computes the normalized average weights and a normalized weight interval over the directly assigned weights or weights output by taking expected utilities in the lotteries and sure values in the assignments based on tradeoffs. In any case, such normalized average weights taken from the DM's imprecise responses will be denoted by $k_{i}^{P}$. The superscript $P$ in $k_{i}^{P}$ means precise weights, and note that the DM can specify identical interval endpoints, which means that the DM is considering a precise value, i.e., $k_{i}^{L}=k_{i}^{U}=k_{i}^{P}$. 


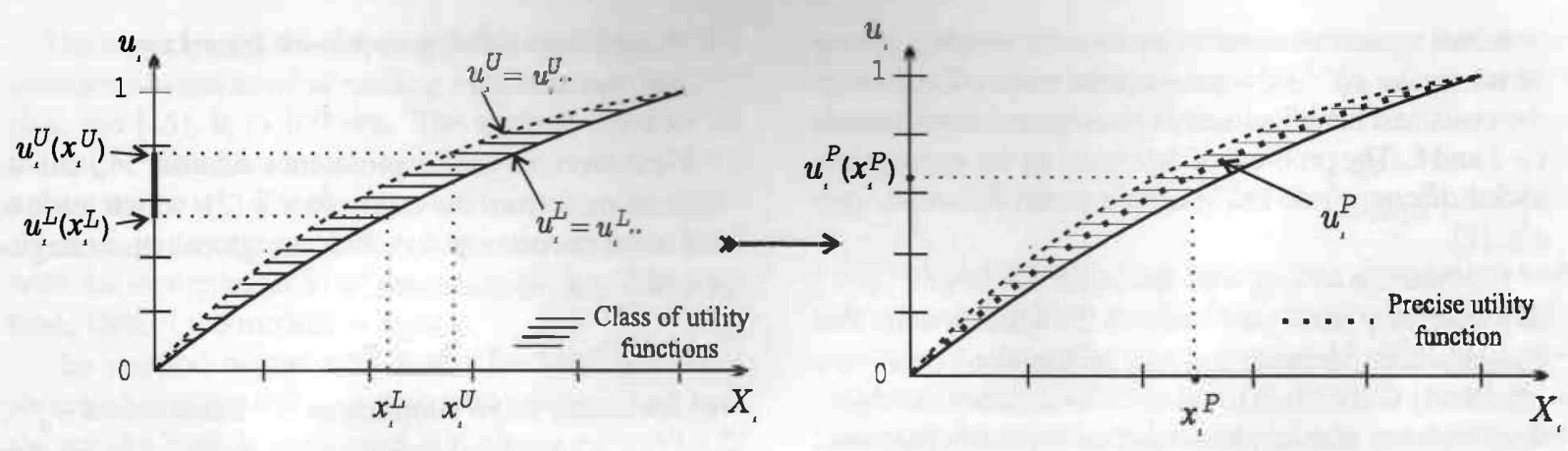

Fig. 3. Utility function class and precise utility function for attribute $X_{i}$.

Assuming the additive independence condition [16], or an approximation [20] and [27], we have a global utility function of the form $u(x)=\sum_{i=1}^{n} k_{i}^{P} u_{i}^{P}\left(x_{i}\right)$, where $x_{i}$ is a specific amount of $X_{i}$. However, due to the imprecision concerning the consequences in our approach, the utility for strategy $S^{q}$ is given by a vector of utility intervals

$$
\begin{aligned}
& \left(\left[\sum_{i=1}^{n} k_{i}^{L} u_{i}^{L}\left(x_{i q}^{L 1}\right), \sum_{i=1}^{n} k_{i}^{U} u_{i}^{U}\left(x_{i q}^{U 1}\right)\right], \ldots,\right. \\
& \left.\left[\sum_{i=1}^{n} k_{i}^{L} u_{i}^{L}\left(x_{i q}^{L T}\right), \sum_{i=1}^{n} k_{i}^{U} u_{i}^{U}\left(x_{i q}^{U T}\right)\right]\right)
\end{aligned}
$$

(provided that the utility functions are increasing), each interval associated with each time period $t$. Now, our problem is to provide a method for ranking the strategies characterized by vectors of utility intervals, where a possible approach is based on the use of discount factors.

\section{Discounting over time}

How to trade off the present and the future is a difficult question, and the classical economic answer is to use exponential discounting. There has been a lengthy discussion on this important point, where the prevailing tendency in normative economic theory has been to demand a constant discount rate. However, descriptive research shows that few people value the future in this way. Cognitive theories claim that this is because people fail to picture the future adequately and are averse to waiting. Psychological research has been carried out to investigate how people discount various commodities over time and what affects their discount rate. In [2] there is an interesting discussion about the issues inherent in discounting non-market goods, arguing that it is unreasonable to do so. Thus, we could say that the research shows that discount rates depend on the decision context and framing. Briefly, behavioral research suggests that discount rates depend on the time until the outcome. People often have discount rates that decrease as the time to the outcome increases and, even so, normative theories of intertemporal choice assume that discount factors are and should be constant. The axiom states that the preference for two outcomes is independent of the time when the outcomes are evaluated. Therefore, the preference should depend only on the absolute time difference between the outcomes. But some authors claim that this does not agree with the value judgements exhibited, and there are persuasive reasons why it should. Hyperbolic discount rates [1] and [11], do allow DMs to attach more importance to the very long-term future than constant discount rates do. This is something that should be explored.

In our context with imprecise consequences, the discounted utility $u\left(S^{q}\right)$ of an outcome stream (1) associated with a strategy $S^{q}$ for a set of attributes $\left(X_{1}, \ldots, X_{n}\right)$ and $T$ time periods, given the stationarity condition combined with the above additive independence, is the vector

$$
\begin{gathered}
{\left[\sum_{i=1}^{n} \sum_{t=1}^{T} k_{i}^{L} \rho_{i}^{t-1} u_{i}^{L}\left(x_{i q}^{L t}\right),\right.} \\
\left.\sum_{i=1}^{n} \sum_{t=1}^{T} k_{i}^{U I} \rho_{i}^{t-1} u_{i}^{U}\left(x_{i q}^{U t}\right)\right]
\end{gathered}
$$

where the first component corresponds to the lower endpoints of the imprecise consequences and the second component to the upper ones. Thus, the discounting method assigns the timing weights $\rho_{i}=\left(1 /\left(1+r_{i}\right)\right)$ to each attribute for each future cost and benefit according to the year $t=1, \ldots, T$, during which the cost or benefit occurs. In this constant-discounting model, the timing weights form a geometric sequence, that is, $\rho_{i}^{t-1}=\left(1 /\left(1+r_{i}\right)\right)^{t-1}$ for $t=1,2, \ldots$, where the pa- 
rameters $r_{i}$ are the social discount rates and the expression $1+r_{i}=\rho_{i}^{t-1} / \rho_{i}^{t}$ represents the trade-offs between the costs and benefits in any two adjacent time periods $t-1$ and $t$. The problem of determining the appropriate social discount rate has received much attention, see, e.g. [2].

For medium to long time spans, i.e. for large $T, \rho_{i}^{t-1}$ becomes very small as $t$ tends to $T$, which means that consequences towards the end of the timestream are effectively discounted to zero. To overcome this objection, we have also implemented an approach proposed in [10] and [11], which suggest a varying discount rate that could devaluate the future at a much slower rate. This is the proportional-discounting model, where the timing weights form a linear fractional sequence, that is, $\left(b_{i} /\left(b_{i}+t\right)\right)^{r_{i}}$, where $b_{i}>0$ is the temporal mid-value for attribute $X_{i}$, that is, the point in the future given half the weight of the present, and $-\infty<r_{i}<\infty$ reflects how the ratio of the outcomes is related to the ratio of the time effects. So, if the DM is timing averse, the utility of a future stream of imprecise multiattributed outcomes is

$$
\begin{gathered}
{\left[\sum_{t=1}^{T} k_{1}^{L} u_{1}^{L}\left(x_{1 q}^{L t}\right)+\sum_{i=2}^{n} \sum_{t=1}^{T}\left(\begin{array}{c}
b_{i} \\
b_{i}+t
\end{array}\right)^{r_{i}} k_{i}^{L} u_{i}^{L}\left(x_{i q}^{L t}\right),\right.} \\
\left.\sum_{t=1}^{T} k_{1}^{U} u_{1}^{U}\left(x_{1 q}^{U t}\right)+\sum_{i=2}^{n} \sum_{t=1}^{T}\left(\begin{array}{c}
b_{i} \\
b_{i}+t
\end{array}\right)^{r_{i}} k_{i}^{U} u_{i}^{U}\left(x_{i q}^{U t}\right)\right]
\end{gathered}
$$

Note that the values in expression (4) decay to 0 as time tends to infinity slower than (3) and, therefore, this approach attaches relatively more importance to longterm outcomes. The model focuses on the ratio between two time periods and not on the absolute difference in their time. To use model (4), it is necessary to determine $b_{i}$ and $r_{i}$ for each attribute $X_{i}$ for the DM, see [10] and [11].

The next thing to be modelled in the DSS is the case where the DM assumes that the assessment of discount factors is a difficult task for certain scenarios, and an alternative approach must be provided. In this situation, we consider for each strategy $S^{q} \in \mathrm{S}$, the vector of utility intervals (2) for each time period $t$ and, as before, the problem involves choosing the best strategy now based on that vector.

In short, we have that each strategy $S^{q}$ will be characterized in all the above cases by a vector of utility intervals

$$
\mathbf{u}\left(S^{q}\right)=\mathbf{u}_{q}=\left(\left[u_{q}^{L 1}, u_{q}^{U 1}\right], \ldots,\left[u_{q}^{L T}, u_{q}^{U T}\right]\right)
$$

of dimension 1 or $T$, depending on the case. Next, we provide a problem-solving approach to solve this complex vector optimization problem.

\section{A problem-solving approach based on approximation}

First we note that a preference relation $\succ_{\mathbf{u}}$ can be defined on $S$ from the utility vector (2), which leads to a dominance principle, defined as: given two strategies $S^{q}, S^{m} \in S$, we have that

$$
S^{q} \succ_{\mathbf{u}} S^{m} \Leftrightarrow \mathbf{u}_{q} \geqslant \mathbf{u}_{m},
$$

which means that $S^{q}$ dominates $S^{m}$ if and only if $u_{q}^{L t} \geqslant$ $u_{m}^{U t}, \forall t$, with at least one strict inequality. The relation $\succ_{\mathbf{u}}$ is a strict partial order on $S$ and, hence, we state the vector optimization problem as

$$
\begin{array}{cl}
\max & \mathbf{u}\left(S^{q}\right) \\
\text { s.t. } & S^{q} \in \mathrm{S}
\end{array}
$$

A natural concept is that $S^{q} \in \mathrm{S}$ is an imprecise utility efficient vector strategy if there is no $S^{m} \in \mathrm{S}$ such that $S^{m} \succ_{\mathbf{u}} S^{q}$ or, equivalently, $\mathbf{u}_{m} \geqslant \mathbf{u}_{q}$. This strategy set will be called imprecise timestream utility efficient vector set and denoted by $\varepsilon_{I}(S, \mathbf{u})$. This leads to the problem "Given $S$ and $\succ_{\mathbf{u}}$, find $\varepsilon_{I}(S, \mathbf{u})$ ". Clearly, if the set $\mathrm{E}_{I}(\mathrm{~S}, \mathbf{u})$ had a single element $S^{q}$, it would be the most preferred strategy for the decision-making problem. However, this is not the case in most real problems, because $\varepsilon_{I}(S, \mathbf{u})$ could contain a lot of ele ments. Thus, our problem should be restated as "select a single element from the set $\varepsilon_{I}(S, \mathbf{u})$ ". One way to solve this decision-making problem, favored by behavioral approaches, will be possible if the DM is able to reveal more information on his/her preferences to provide additional structural assumptions and get a subset of $\varepsilon_{I}(S, \mathbf{u})$. For this purpose, we provide an interactive method, based on multiobjective simulated annealing, to progressively build an approximation set to $\varepsilon_{I}(S, \mathbf{u})$ in collaboration with the DM, which is valid for any of the above settings and based on vectorial optimization for the imprecise utility vectors with different dimensions depending on the model used.

\section{Interactive simulated annealing-based search}

The idea of the method we propose is based on the interaction with set $S$, which is associated with the respective set of vectors of utility intervals (1-dimensional for cases (3) and (4) and $T$-dimensional for (2)). We then generate an approximation set $A(S, \mathbf{u})$ of the imprecise timestream utility efficient vector set $E_{I}(S, \mathbf{u})$, i.e., solutions not dominated by any other considered solution. 
The underlying idea of this evaluation method, called interactive simulated annealing based on approximation, see [15], is as follows. The method is based on the set $S$, where each element $S^{q}$ is associated with a utility vector $\mathbf{u}^{q}$. We then generate an approximation set $A(S, \mathbf{u})$ (u represents the utility vectors associated with the strategies in $S$ ) of the set $E_{I}(S, \mathbf{u})$. Then, the basic idea of the method is simple.

The method begins with a first iteration providing an initial strategy $S^{0} \in S$ drawn at random, and thus the set $A(S, \mathbf{u})$ is initialized containing only $S^{0}$. In the following iterations, another strategy $S$ from the neighborhood of the solution in the current iteration is considered, and $S$ is accepted if it is not dominated by the strategies currently in the approximation set. In this case, we add $S$ to the set $A(S, \mathbf{u})$ and throw out any solution in $A(S, \mathbf{u})$ dominated by $S$. On the other hand, if $S$ were dominated by any element in A $(S, \mathbf{u})$, we would continue considering $S$ for the next iteration with a given probability, known as acceptance probability. In this way, according to the movement in the iterations through the space, we simultaneously build the set $A(S, \mathbf{u})$.

The acceptance probability used in our context is the multiobjective product rule, see [7]. A weighting vector $\lambda$ is needed to define such probability. Now, let $A(S, \mathbf{u}, \lambda)$ be the potentially efficient utility solutions set generated by simulated annealing using the weighting vector $\lambda$. Note that by controlling the weights, we shall be able to increase or decrease the acceptance probability of new solutions, which means selecting a certain set of weights that could lead us towards a particular region formed by the potentially efficient utility solutions.

The procedure for obtaining a good approximation $A(S, \mathbf{u})$ of the set $E_{I}(S, \mathbf{u})$, is as follows: Taking a weighting vector set $W$, generated in an extensively diversified way, we get, for each $\lambda$, a list $A(S, \mathbf{u}, \lambda)$ that contains the potentially efficient utility solutions in the direction induced by it. Finally, we need to filter the set $\bigcup_{\lambda \in W} A(S, \mathbf{u}, \lambda)$ by pairwise comparison to output the non-dominated solutions. This filtering process will be denoted by $\Lambda$, in such a way that $A(S, \mathbf{u})=$ $\wedge \cup_{\lambda \in W A}(S, \mathbf{u}, \lambda)$.

On the other hand, a set of minimum satisfaction levels $\varepsilon_{t}$ must be provided by the DM for the different attributes, which represent the bounds within which each one of the utilities of the different components satisfies him/her individually. Through an interactive process, I will modify these satisfaction levels according /her preferences in response to the information aine $\mathrm{d}$ as follows.
First, the minimum satisfaction levels are initialized and the elements of an initial weighting vector set $W^{0}$ are drawn at random from the set

$$
\left\{\lambda \in \mathbb{R}^{T}: \lambda_{t} \in[0,1] \forall t \text { and } \sum_{t=1}^{T} \lambda_{t}=1\right\}:
$$

$50 \%$ of which come from the uniform distribution and the remaining from the Weibull distribution, see [26]. Moreover, the weighthing vectors $\mathbf{1}^{j}$ with all components equal to 0 but the $j^{\text {th }}$ position equal to 1 are also included in this set. Thus, an initial list $L_{0}=\wedge \cup_{\lambda \in W^{0}} A(S, \mathbf{u}, \lambda)$ is generated as a result of the filtering operation considered above.

Next, in each iteration, the list output in the previous one, $L_{M-1}$, is presented to the DM, who modifies the minimum satisfaction levels according to his/her preferences on this list. Then, a new restricted list of weighting vectors $W^{M}$ is generated removing the weights that become useless with respect to the new satisfaction levels and where a new weighting vector is also introduced so that an empty set of weights is not output (when the DM's satisfaction levels are too strong), see [15].

Now, we perform multiobjective simulated annealing with the weighting vectors in $W^{M}$ in order to output the new list of solutions

$$
\begin{aligned}
L_{M}= & \bigwedge\left\{L_{M-1} \cup\left\{\mathrm{A}(\mathrm{S}, \mathbf{u}, \lambda), \lambda \in \mathbf{W}^{\mathbf{M}}\right\}\right\} \\
& \cap\left\{S_{q}: u_{q}^{P t} \geqslant \varepsilon_{t} \forall t\right\} .
\end{aligned}
$$

Note that the minimum satisfaction levels are also used to limit the input of solutions in $L_{M}$.

The interactive simulated annealing ends when the $\mathrm{DM}$ is completely satisfied with a solution or set of solutions of $L_{M}$.

\section{Sensitivity analysis}

Sensitivity analysis (SA), which essentially invo tue examining changes in the ranking as a function of the input parameters (weights, utilities and strategy $c_{0}$ quences) varying within a reasonable range, can further insight into the robustness of the recomm tions. Some types of sensitivity analysis are deocined in [14], which introduces a framework for sensitivity analysis in multiobjective decision making. SA is usually performed by changing the weights or utilitiobserving their impact on the ranking of altem inHence, if the DM makes a change to an average non ized weight or a bound of a normalized weight interval, the system takes cares of how these changes are $r$ 
gated through the objective hierarchy and automatically recalculates the list $L_{0}$ of potentially optimal solutions to run a new interactive problem-solving process. It also recalculates the overall utility for each strategy and the resulting ranking based on the precise values for the weights, individual utilities and consequences.

Another way of performing SA involves assessing the interval in which the weight for a specific objective can vary, maintaining a constant ratio among the other weights, without affecting the overall strategy ranking. Suppose that we currently have a ranking of the given strategies and the DM chooses a node or leaf of the objective tree with an associated weight. The system calculates the weight interval for this node/leaf, taking into account the updated weights for the objectives stemming from its predecessor, so that the ranking based on the precise assignments does not change. In other words, if the weight is changed and the new value is within the range, then the ranking will not change. However, if the new value is not within the range, the new ranking will be different to the previous one.

Finally, the system runs simulation techniques for SA, see [3] and [4]. This kind of sensitivity analysis allows for simultaneous changes in weights and generates results that can be easily statistically analyzed to provide more insights into the multiattribute model recommendations. We propose selecting weights at random using a computer simulation program so that the results of many combinations of weights, including a complete ranking, can be explored efficiently.

Three general classes of simulation are implemented: random weights, rank order weights and response distribution weights. They are described briefly below.

a) Random weights: As an extreme case, attribute weights are generated completely at random. This approach implies no knowledge whatsoever of the relative importance of attributes. In many multicriteria settings, the scores of the strategies significantly limit the subset of potential rankings.

b) Rank order weights: Randomly generating weights while preserving an attribute rank order places substantial restrictions on the domain of possible weights that are consistent with the DM's judgement of attribute importance. Therefore, the results from the rank order simulation may provide more meaningful results. The DM can introduce the rank order for all or only some of the attributes of the problem.

c) Response distribution weights: The third type of simulation-based sensitivity analysis recognizes that the weight assessment procedure is subject to variation. For a single DM, this variation may be in the form of response error associated with weight assessment. Thus, whereas in the first class of simulation, attribute weights were randomly assigned values between 0 and 1 (taking into account that the sum of the whole is 1 ), attribute weights are now randomly assigned values taking into account the attribute normalized weight intervals provided by the DM through the weights assignment methods.

Once the simulation has been run, the system computes several statistics about the rankings of each strategy, like mode, minimum, maximum, mean, standard deviation and the 25 th, 50 th and 75 th percentiles. This information can be useful for discarding some possible strategies, aided by a display which presents a multiple boxplot for the strategies.

\section{An application to the restoration of an aquatic ecosystem}

In this section, we describe the application of the DSS to the analysis of a set of remedial strategies for restoring Lake Kozhanovskoe, located in the Bryansk region in Russia, which was heavily contaminated with ${ }^{137} \mathrm{Cs}$ after the Chernobyl accident in 1986. This complex decision-making problem was studied in a simpler context (taking a static approach, precise inputs and certainty over the consequences) using the MOIRA system, see [9]. In 1998, Lake Kozhanovskoe was classed as a radio-ecological reservation, and fishery was officially forbidden because of the high levels of fish contamination with ${ }^{137} \mathrm{Cs}$. The population around the lake was evacuated, as its area belonged to the population evacuation zone as the levels of contamination with ${ }^{137} \mathrm{Cs}$ were rather high $\left({ }^{137} \mathrm{Cs}\right.$ fallout on the lake was about $600000 \mathrm{~Bq} / \mathrm{m} 2$ ). However, many residents continued to live at villages near the lake, and fish caught in lake Kozhanovskoe was a predominant food of the local residents even 10 years after the Chernoby 1 accident.

In spite of the ban on fishing proposed by the expert assessment, 20-30 families were still engaged in fishing. In addition, amateur fishermen often went fishing to the lake from neighbouring districts and caught fish for the most part with fishing rods. Consumption of fish by the fishermen and members of their families could be as great as $80 \mathrm{~kg}$ a year per person, while. on average, the residents of this area consumed $20 \mathrm{~kg}$ 


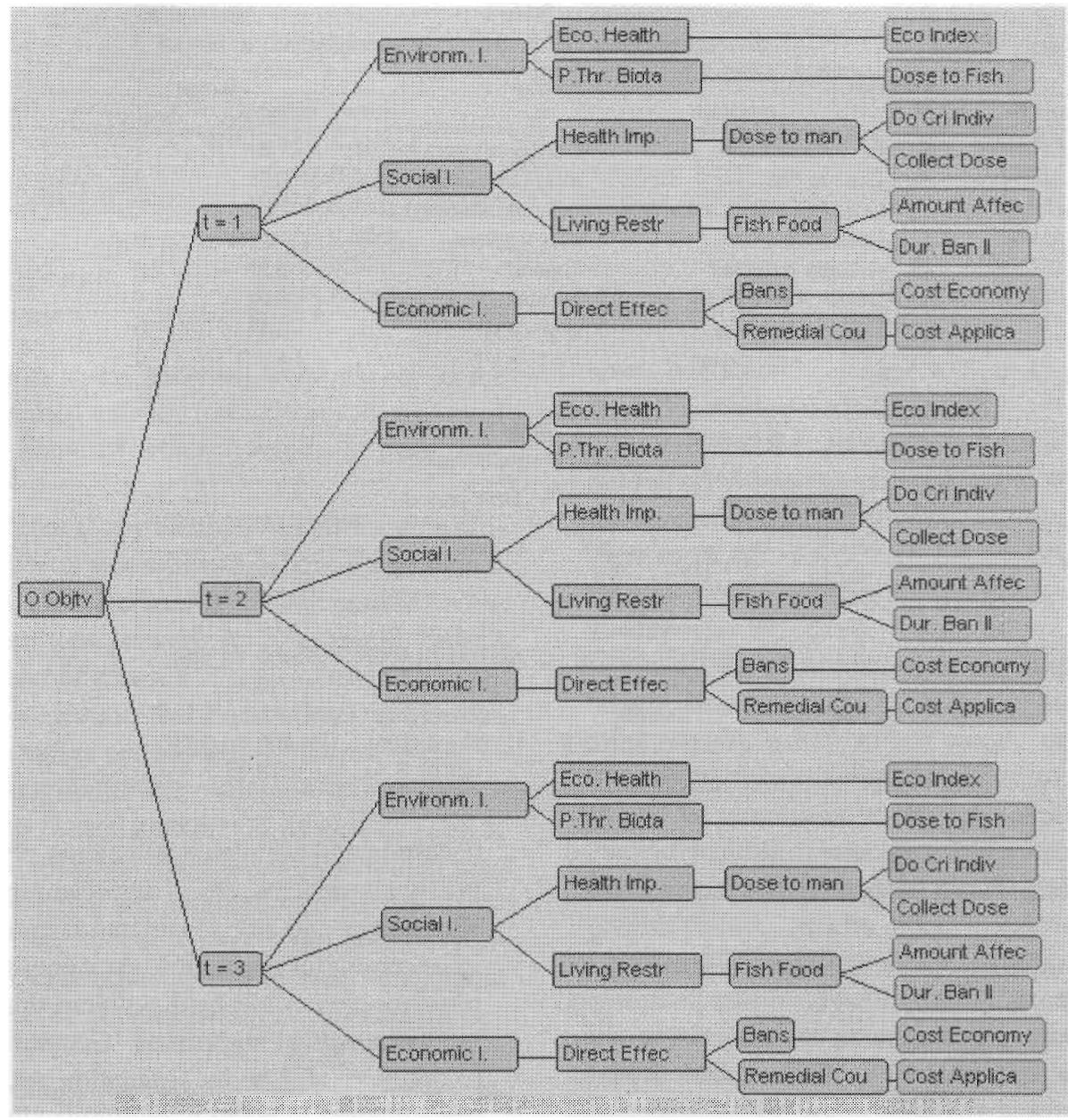

Fig. 4. Time-dependent objective hierarchy for the restoration of Lake Kozhanovskoe.

of fish annually. In 1998 , the concentration of ${ }^{137} \mathrm{Cs}$ in predatory species of fish from Lake Kozhanovskoe remained high and was, on average, $20-25 \mathrm{kBq} / \mathrm{kg}$, fresh weight, whereas it was 6-10 kBq/kg, fresh weight in non-predatory species. Therefore, the intervention strategies were mainly oriented at reducing either fish contamination or the amount of fish consumed by the population.

In this context, the above decision system supports the DM's choice of the best restoration decision alternative. An objective hierarchy was built for this decision-making problem, which intended to provide the grounds for the description and evaluation of the hypothetical restoration alternatives for the scenario in question. Relevant objectives, as well as the attributes associated with the lowest-level objectives of the hierarchy, were established in the DSS. Figure 4 shows the tree diagram for this problem, with the same attribute set for each one of the three time periods proposed.
The attributes associated to the lowest-level objectives are:

1. Ecosystem Index $\left(X_{1}\right)$ The Ecosystem Index is a measure of the ecosystem health in aquatic environments. It summarises the state of important ecosystem variables affecting phytoplankton, benthos and fish.

2. Dose to Fish $\left(X_{2}\right)$. An assessment of the absorbed radiation dose received by fish as a consequence of the radioactive contamination of the aquatic ecosystem.

3. Dose to Critical Individual $\left(X_{3}\right)$. The effective dose received by individuals belonging to a critical group living in the area, drinking water, and eating aquatic food and terrestrial food irrigated with water from the contaminated water body.

4. Collective Dose $\left(X_{4}\right)$. The collective effective dose received by all the population through all 


\begin{tabular}{llcc}
\hline Attribute & Measure & \multicolumn{2}{c}{ Range } \\
\cline { 3 - 4 } & & Worst $x_{i *}$ & Best $x^{*}$ \\
\hline$X_{1}:$ Ecosystem Index & LEI (Ecosystem Index) & 5 & 1 \\
$X_{2}:$ Dose to Fish & mGy (MiliGray) & $10^{4}$ & 0 \\
$X_{3}:$ Dose to Critical Individual & mSv (miliSievert) & 500 & 0 \\
$X_{4}:$ Collective Dose & mSv $\times$ person & $12 \times 10^{4}$ & 0 \\
$X_{5}:$ Amount Affected & Tonnes & $10^{4}$ & 0 \\
$X_{6}:$ Duration of Ban II & Months & 120 & 0 \\
$X_{7}:$ Cost to Economy & Euros $\times 10^{2}$ & $10^{8}$ & 0 \\
$X_{8}:$ Cost of Application & Euros $\times 10^{2}$ & $2 \times 10^{8}$ & 0 \\
\hline
\end{tabular}

Table 2

Outcome stream for $S^{6}$

\begin{tabular}{lccc}
\hline \multicolumn{4}{c}{ Time period $t$} \\
& 1 & 2 & 3 \\
\cline { 3 - 4 }$X_{1}$ & {$[1.31,1.40]$} & {$[1.30,1.42]$} & {$[1.28,1.43]$} \\
$X_{2}$ & {$[1700,1820]$} & {$[1660,1800]$} & {$[1590,1750]$} \\
$X_{3}$ & {$[10.9,11.3]$} & {$[10.7,11.2]$} & {$[10.3,11.0]$} \\
$X_{4}$ & {$[61000,63000]$} & {$[60700,62800]$} & {$[60400,61300]$} \\
$X_{5}$ & {$[540,600]$} & {$[150,165]$} & {$[0,0]$} \\
$X_{6}$ & {$[60,60]$} & {$[30,30]$} & {$[0,0]$} \\
$X_{7}$ & {$[342000,367000]$} & {$[12000,48300]$} & {$[11000,54500]$} \\
$X_{8}$ & {$[0,0]$} & {$[0,0]$} & {$[0,0]$} \\
\hline
\end{tabular}

the exposure pathways due to the contaminated water body. It is a measure of the increased risk of serious latent health effects.

5. Amount Affected $\left(X_{5}\right)$. In the case of restrictions to fish consumption and food industry processing, the amount of fish affected by restrictions.

6. Duration of Ban $I\left(X_{6}\right)$. In the case of restrictions to fish consumption and food industry processing, the duration of the restrictions.

7. Cost to Economy $\left(X_{7}\right)$. The direct economic impact of the restrictions, either in terms of the cost of the food affected by bans or in terms of the production lost (e.g., share of Gross Domestic Product Lost).

8. Cost of Application $\left(X_{8}\right)$. In the case of remedial countermeasures (physical or chemical), this represents the direct cost of the application: manpower, consumables, equipment needed for the application, management of wastes generated, etc.

Table 1 shows these attributes as well as their measurement units and the respective ranges provided for the strategies analyzed later.

Several intervention strategies or alternatives must be created to reflect what could happen once the scenario has been settled. Expected conditions occur, based on the available information, but as the multiattribute consequences of each possible strategy are assumed to be uncertain and Simulated Annealing generates stochastically alternatives, the system can randomly generate alternatives and any considered feasible will be taken into account for evaluation. As a consequence, we will possibly have a NP-hard problem that can be solved by mathematical algorithms. Generally, the only way to optimize will be by simulation with the aid of the implemented interactive multiobjective simulated annealing. As shown with this approach, DM can change several parameters to interactively reduce the imprecise utility efficient set until a final alternative is obtained.

In this example, we consider six strategies whose descriptions are:

$S^{1}$ : No Action. Natural evolution of the situation without intervention.

$S^{2}$ : Potash Treatment. Reduction of aquatic organism uptake by potash treatment of aquatic ecosystems contaminated by radiocesium.

$S^{3}$ : Fertilization. Tonnes of fertilizer added to the lake to increase biomass (biological dilution).

$S^{4}$ : Lake Liming. Reduction of radionuclide remobilisation from sediments.

$S^{5}$ : Sediment Removal. $6 \mathrm{~km}^{2}$ of sediments removed from the lake down to depth of $5 \mathrm{~cm}$.

$S^{6}$ : Automatic Food Bans. Automatic fish consumption ban when ${ }^{137} \mathrm{Cs}$ content in fish is greater than $1000 \mathrm{~Bq} / \mathrm{kg}$. 
Table 3

Vector utilities $\mathbf{u}_{i}, \boldsymbol{i}=1, \ldots, 6$, for the constant-discounting model with $r=5 \%$

$\begin{array}{cc}\text { Strategy } & \text { Utility vector } \\ S^{1} & {[0.223,0.547]} \\ S^{2} & {[0.449,0.846]} \\ S^{3} & {[0.163,0.431]} \\ S^{4} & {[0.189,0.468]} \\ S^{5} & {[0.223,0.521]} \\ S^{6} & {[0.295,0.689]}\end{array}$

Alternatives must be analyzed with respect to the attributes associated with the lowest-level objectives of the hierarchy, some of which (perhaps all) are time dependent.

To show the underlying ideas of the optimization process in detail, we only consider the above six strategies $S^{j}, j=1, . ., 6$, characterized by the imprecise outcome streams for three time periods. As an example, Table 2 shows the impact of strategy $S^{6}$, given as an outcome stream, where we assume that the time interval between each two consecutive periods is five years.

The system computes the respective utility interval for each strategy in each time period, as shown in Fig. 5, where the strategies are ranked according to their precise utilities, weights and consequence values. Note that the utility intervals could get wider with the passage of time, if there is an increase in uncertainty about the consequence, or get narrower if the uncertainty decreases.

Thus, for example, strategy $S^{1}$ is characterized by the utility interval vector $(1)$

$$
([0.250,0.759],[0.198,0.0 .559],[0.190,0.546]) .
$$

Assuming a constant-discounting model to be valid, where the DM assigns the same discount rate $r=5 \%$ for all the attributes, which leads to a discount factor $\rho=1 /(1+0.05) \simeq 0.9524$, where the discount rates for time periods 2 and 3 are 0.9071 and 0.7836 , respectively, then, the vector utilities associated with each strategy are given in Table 3.

We see that $S^{2}$ dominates $S^{3}$ and, thus, the imprecise utility efficient vector set is $\varepsilon_{I}(\mathrm{~S}, \mathbf{u})=$ $\left\{S^{1}, S^{2}, S^{4}, S^{5}, S^{6}\right\}$. Although this is a very small set of strategies for applying the approximation approach based on interactive multiobjective simulated annealing, which has been developed to solve complex problems, we consider application to our example to illustrate its use. Thus, if we apply the problem-solving procedure with default parameter values, we get the approximation set $\mathrm{A}(\mathrm{S}, \mathbf{u}, \lambda)=\left\{S^{1}, S^{2}, S^{6}\right\}$. Now, by changing the parameters and the minimum satisfaction
Table 4

Vector utilities $\mathbf{u}_{i}, i=1, \ldots, 6$, for the proportional-discounting model with $b=0.5$ and $r=1$

\begin{tabular}{cc} 
Strategy & Utility vector \\
\hline$S^{1}$ & {$[0.322,0.641]$} \\
$S^{2}$ & {$[0.519,0.901]$} \\
$S^{3}$ & {$[0.224,0.532]$} \\
$S^{4}$ & {$[0.245,0.636]$} \\
$S^{5}$ & {$[0.302,0,703]$} \\
$S^{6}$ & {$[0.412,0.783]$} \\
\hline
\end{tabular}

levels interactively, we get $A(S, \mathbf{u}, \lambda)=\left\{S^{2}\right\}$, where $S^{2}$ is the most preferred solution.

Now, if the DM chooses the proportional-discounting model, providing the values $b=0.5$ and $r=1$ for all the attributes, the vector utilities are shown in Table 4. Here $\varepsilon_{I}(\mathrm{~S}, \mathbf{u})=\left\{S^{1}, S^{2}, S^{3}, S^{4}, S^{5}, S^{6}\right\}=\mathrm{S}$, but the strategy we get after applying interactive simulated annealing is $S^{6}$.

Finally, the system incorporates sensitivity analysis, which allows the DM to make a change to an endpoint weight, a normalized weight or a utility function. Then, the system takes care of how these changes should be propagated in the time-dependent objective hierarchy and automatically recalculates the utility vector for each strategy and the resulting ranking based on precise values.

If the DM runs simulations, the system computes several statistics about the rankings of each strategy, like mode, minimum, maximum, mean, standard deviation and the 25 th, 50 th and 75 th percentiles. This information can be useful for discarding some possible strategies, aided by a display that presents a vertically grouped boxplot for the strategies.

Thus, the DSS includes a multiparametric sensitivity analysis facility to gain additional insight into the strategy ranking, which demonstrates that these optimization problems will be more tractable if the temporal domain is used to take into account their effects.

\section{Conclusions}

We have developed a decision support system for the decision analysis process in systems engineering based on imprecise inputs concerning weights for all the objectives of the value tree, individual utilities for each attribute and consequences of the available strategies, and supported by a dynamic (objective tree) structure, which is intended to provide the grounds for the description and evaluation of hypothetical management strategies or decision alternatives for the different scenarios to be considered by the DM. Relevant objectives 


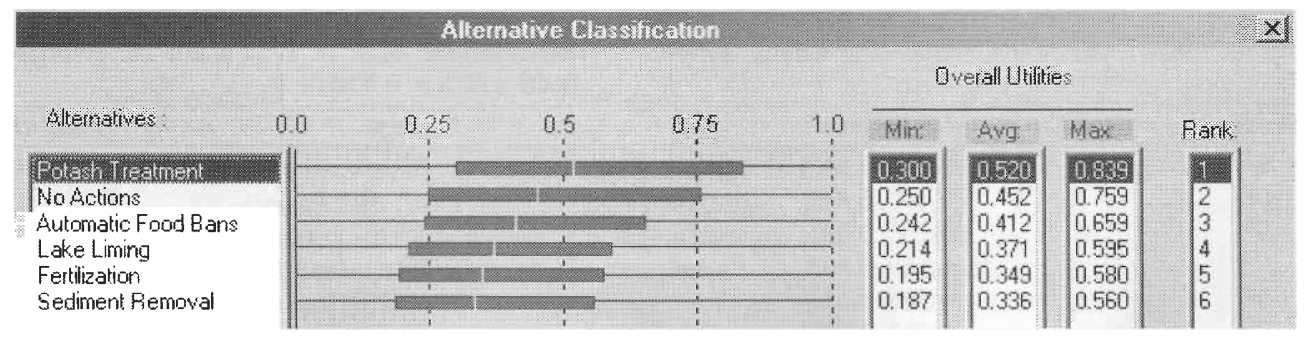

Time period 1

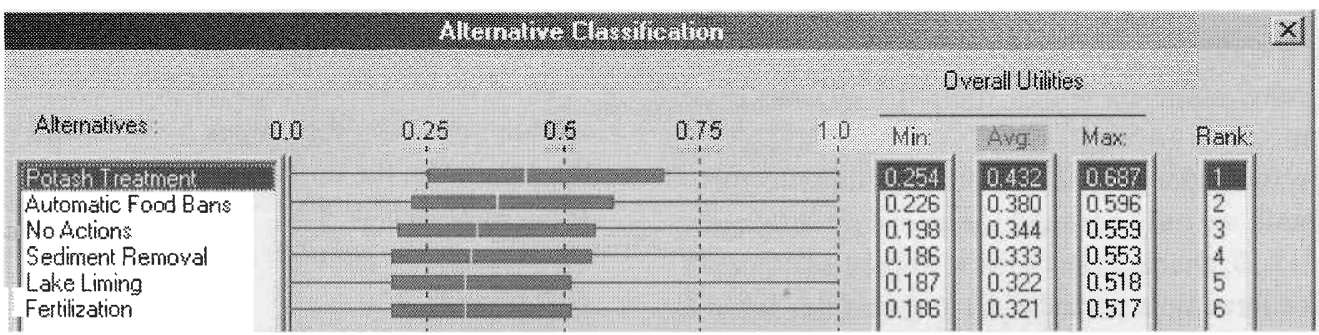

Time period 2

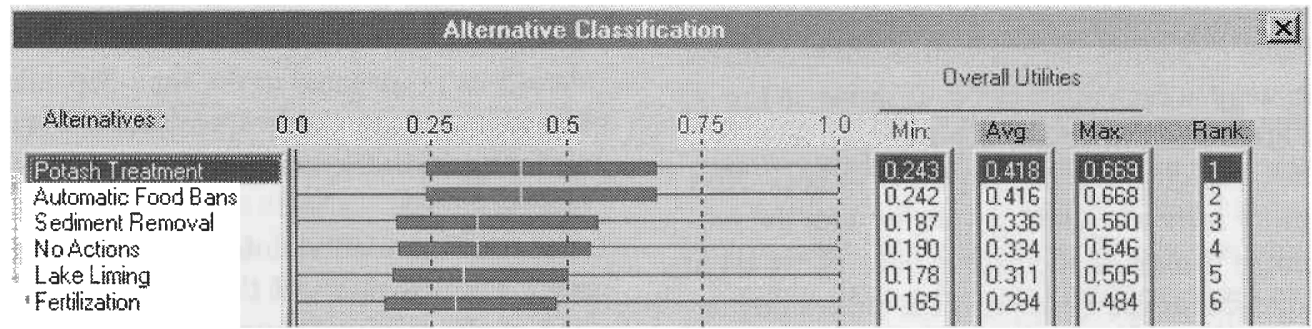

Time period 3

Fig. 5. The utility intervals and the ranked strategies based on their precise utilities for each time period.

as well as the attributes associated with the lowest-level objectives of the hierarchy are created in the DSS. Scenarios need to contain enough technical information for them to be realistic, while they should not be so technical as not to be understandable. The alternatives must be analyzed with respect to the attributes associated with the lowest-level objectives of the hierarchy, some of which (maybe all) are time dependent. Next, the DM will have to assess the imprecise utility functions, scaling constants and discount factors, if deemed appropriate, which will be used to provide the inputs for the corresponding model, associating each strategy with a vector of utility intervals, whose dimension will be equal to the number of time periods considered, so we will have only one utility interval for the static case. The application of interactive multiobjective simulated annealing leads to the determination of the best or, at least, a small enough set of satisfactory strategies, from which the DM could choose the final strategy more easily by changing satisfaction levels or using a multiparametric sensitivity analysis. This means that the user can gain additional insight into the ranking of the alternatives. This demonstrates that this kind of optimization problems may be more tractable by using the temporal domain to take into account their effects.

\section{List of symbols}

S: Set of available strategies

$P$ : Precision

$\rho_{i}$ : Timing weight

$r_{i}$ : Social discount rate

$b_{i}$ : Temporal mid-value for attribute $X_{i}$

$\succ_{\mathbf{u}}$ : Preference relation based on the vectorial utility

$\mathbf{u}$

$\varepsilon_{I}(S, \mathbf{u})$ : Imprecise timestream utility efficient vector set 
$\lambda$ : Weighting vector

A $(S, \mathbf{u})$ : Approximation set

$A(S, \mathbf{u}, \lambda)$ : Approximation set using weighting vector $\lambda$

$\bigwedge$ : Filtering operator

$W^{j}$ : List of weighting vectors in the $j-t h$ iteration

$L_{j}$ : List of solutions in the $j-t h$ iteration

$\varepsilon_{t}$ : Minimum satisfaction levels

\section{Acknowledgement}

This paper was supported by the Ministry of Science and Technology project DPI 2001-3731.

\section{References}

[1] M. Ahlbrecht and M. Weber, Hyperbolic Discount Models in Prescriptive Theories of Intertemporal Choice, Zeitschrift fir Wirtschaft 115 (1995), 535-568.

[2] E. Atherton and S. French, Issues in Supporting Intertemporal Choice, in: Essays in Decision Making, M. Karwan, J. Spronk and J. Wallenius, eds, Springer-Verlag, 1997, pp. 135-156.

[3] J. Butler, J. Jia and J. Dyer, Simulation Techniques for the Sensitivity Analysis of Multi-Criteria Decision Models, European Journal of Operational Research 103 (1997), 531-546.

[4] J.S. Dyer, T. Edmunds, J.C. Butler and J. Jia, A Multiattribute Utility Analysis of Alternatives for the Disposition of Surplus Weapons-Grade Plutonium, Operations Research 46(6) (1998), 749762.

[5] P.H.Farquhar, Utility Assessment Methods, Management Science 30 (1984), 1283-1300.

[6] P.C. Fishburn, Utility Theory for Decision Making, John Wiley, New York, 1970.

[7] P. Fortemps, J. Teghem and B. Ulungu, Heuristics for Multiobjective Combinatorial Optimization by Simulated Annealing, 11 th International Conference on MCDM, Coimbra, Portugal, 1994.

[8] S. French, Decision Theory: An Introduction to the Mathematics of Rationality, Ellis Horwood, Chichester, 1986.

[9] E. Gallego, A.Jiménez, A. Mateos, T. Sazykina, S. Rios-Insua and M. Windergärd, Application of Multiattribute Analysis Methodologies to the Evaluation of the Effectiveness of Remedial Strategies with the MOIRA System, L. Monte et al., eds, Report RT/AMB/2001/28, ENEA, Roma, 2001, pp. 111-130.

[10] C. Harvey, A Slow Discounting Model for Energy Conservation, Interfaces 22 (1992), 47-60.

[11] C. Harvey, Proportional Discounting of Future Benefits and Costs, Mathematics of Operations Research 20 (1995), 381399.

[12] J.C. Hershey, H.C. Kunreuther and P.J. Schoemaker, Sources of Bias in Assessments Procedures for Utility Functions, Management Science 28 (1982), 936-953.

[13] J.Y. Jaffray, Some Experimental Findings on Decision Mak ing under Risk and their Implications, European Joumal of Operational Research 38 (1989), 301-306.

[14] A. Jiménez, Un Sistema de Ayuda a la Decisión con Asignaciones Imprecisas, Ph D. Dissertation, Madrid Technical University, 2002.

[15] A. Jiménez, S. Ríos-Insua and A. Mateos, Interactive Simulated Annealing for Solving Imprecise Discrete Multiattribute Problems under Risk, Pesquisa Operacional, Special Issue on Data Envelopment Analysis and Multicriteria Decision Aid $\mathbf{2 2}$ (2002), 23-37.

[16] R.L. Keeney and H. Raiffa, Decision with Multiple Objectives, Cambridge University Press, 1993.

[17] J. Kombluth, Dynamic Multicriteria Decision Making, Journal of Multi-Criteria Decision Analysis 1 (1992), 301-309.

[18] A. Mateos, S. Ríos-Insua and E. Gallego, Postoptimal Analysis in a Multi-Attribute Decision Model for Restoring Contaminated Aquatic Ecosystems, Journal of the Operational Research Society 52 (2001), 1-12.

[19] M. McCord and R. de Neufville, Lottery equivalents: Reduction of the Certainty Effect Problem in Utility Assessment, Management Science 32 (1986), 56-61

[20] H. Raiffa, The Art and Science of Negotiation, Harvard University Press, Cambridge, Mass, 1982.

[21] D. Rios Insua, Ambiguity, Imprecision and Sensitivity in: Decision Theory, in Recent Advances in Statistics and Probability, J.P. Vilaplana and M.L. Puri, eds, VSP, 1994, pp. 219-232.

[22] S. Ríos, S. Ríos-Insua, D. Ríos Insua and J.G. Pachón, Experiments in Robust Decision Making, in: Decision Theory and Decision Analysis: Trends and Challenges, S. Ríos, ed., Kluwer, Boston, 1994, pp. 233-242.

[23] D. Rios Insua, E. Gallego, A. Mateos and S. Ríos-Insua, MOIRA: A Decision Support System for Decision Making on Aquatic Ecosystems Contaminated by Radioactive Fallout, Annals of Operations Research 95 (2000), 341-364.

[24] R. Sarin and R. Winkler, Ambiguity and Decision Modelling: A Preference Based Approach, Journal of Risk and Uncertainty 5 (1992), 176-190.

[25] L.J. Savage, The Foundations of Statistics, John Wiley, New York, 1954.

[26] R.E. Steuer and F.W. Harris, Intra-set Point Generation and Filtering in Decision and Criterion Space, Computers and Operations Research 7 (1980), 41-53.

[27] Th.J. Stewart, Robustness of Additive Value Function Method in MCDM, Journal of Multi-Criteria Decision Analysis 5 (1996), 301-309.

[28] J. von Neumann and O. Morgenstern, Theory of Games and Economic Behavior, Princeton University Press, 1947.

[29] R. von Nitzsch and M. Weber, Utility Function Assessment on a Micro-computer: An Interactive Procedure, Annals of Operations Research 16 (1988), 149-160.

[30] M. Weber, Decision Making with Incomplete Information, European Journal of Operational Research 28 (1987), 44-57. 